\title{
Kaon-nuclei interaction studies at low energies (the AMADEUS experiment)
}

K. Piscicchia $\left({ }^{1}\right)\left({ }^{2}\right)(*)$, M. Bazzi $\left({ }^{1}\right)$, C. Berucci $\left({ }^{1}\right)$, A. M. Bragadireanu $\left({ }^{3}\right)$, M. Cargneldi $\left({ }^{4}\right)$, A. Clozza $\left({ }^{1}\right)$, C. Curceanu $\left({ }^{1}\right)$, A. D’Uffizi $\left({ }^{1}\right)$, F. Ghio $\left({ }^{5}\right)$, C. $\operatorname{Guaraldo}\left({ }^{1}\right)$, M. $\operatorname{Iliescu}\left({ }^{1}\right)$, T. Ishimatari $\left({ }^{4}\right)$, P. Levi Sandri $\left({ }^{1}\right)$,

J. Marton $\left({ }^{4}\right)$, S. Okada $\left({ }^{1}\right)$, D. Pietreanu $\left({ }^{1}\right)\left({ }^{3}\right)$, M. Poli $\operatorname{Lener}\left({ }^{1}\right)$, A. Rizzo $\left({ }^{1}\right)$,

A. Romero $\operatorname{Vidal}\left({ }^{1}\right)$, E. Sbardella $\left({ }^{1}\right)$, A. $\operatorname{Scordo}\left({ }^{1}\right)$, D. L. $\operatorname{Sirghi}\left({ }^{1}\right)\left({ }^{3}\right)$,

F. Sirghi $\left({ }^{1}\right)\left({ }^{3}\right)$, H. Tatsuno $\left({ }^{1}\right)$, O. Vazquez Doce $\left({ }^{1}\right)$,

E. Widmann $\left({ }^{4}\right)$ and J. ZMeskal $\left({ }^{4}\right)$

$\left({ }^{1}\right)$ INFN, Laboratori Nazionali di Frascati - Frascati (Rome), Italy

(2) Dipartimento di Fisica, Università degli Studi "Roma Tre" - Rome, Italy

$\left({ }^{3}\right)$ IFIN-HH - Magurele, Bucharest, Romania

${ }^{(4)}$ Stefan-Meyer-Institut für subatomare Physik - Vienna, Austria

$\left({ }^{5}\right)$ INFN, Sezione di Roma 1 and Istituto Superiore di Sanità - Rome, Italy

(ricevuto il 29 Luglio 2011; pubblicato online il 25 Ottobre 2011)

Summary. - The AMADEUS experiment aims to perform dedicated precision studies in the sector of low-energy kaon-nuclei interaction at the DA $\Phi$ NE collider at LNF-INFN. In particular the experiment plans to perform measurements of the so-called deeply bound kaonic nuclei (by stopping kaons in cryogenic gaseous targets ${ }^{3} \mathrm{He}$ and ${ }^{4} \mathrm{He}$ ) and to study the low-energy interaction of negative kaons in various targets. The AMADEUS dedicated setup will be installed inside the KLOE drift chamber.

PACS 13.75. Jz - Kaon-baryon interactions.

PACS 25.80.Nv - Kaon-induced interactions.

PACS 21.65. Jk - Mesons in nuclear matter.

\section{1. - Introduction}

The AMADEUS (Antikaon Matter At DA $\Phi$ NE Experiments with Unraveling Spectroscopy) experiment $[1,2]$ will study the low-energy interactions of kaons with nucleons and nuclei. AMADEUS aims to search for the most fundamental Deeply Bound Kaonic Nuclear States (DBKNS), that are the kaonic dibaryon states $\left(K^{-} p p, K^{-} p n\right)$,

$\left(^{*}\right)$ E-mail: kristian.piscicchia@lnf.infn.it

(C) Società Italiana di Fisica 
produced by stopping $K^{-}$in a ${ }^{3}$ He target and, as a next step, the kaonic tribaryon states $\left(K^{-} p p n, K^{-}\right.$pnn) using a ${ }^{4}$ He target. Also important objectives are the measurements of low-energy cross-sections of charged kaons on $\mathrm{H}$, d, and He (for kaons momentum lower than $100 \mathrm{MeV}$ ) and the study of nuclear interactions of $\mathrm{K}^{-}$in these targets. Kaonic nuclear states will be studied both in the formation process, by means of missing-mass spectroscopy, and in the decay process. As DBKNS are expected to decay into states containing $\Lambda$ and $\Sigma$ hyperons, neutral (neutrons and photons) as well as charged particles are to be detected, with momenta in a wide range. The detection requirements are satisfied by the KLOE detector, which is made of a $4 \pi$ cylindrical drift chamber (DC) and a calorimeter, with an acceptance of $96 \%$.

\section{2. - The dedicated AMADEUS setup}

The AMADEUS setup will be implemented inside the KLOE DC, between the beam pipe (6 $\mathrm{cm}$ diameter) and the DC entrance wall $(50 \mathrm{~cm}$ diameter). Charged kaons produced back to back by $\phi$ decay, will be stopped after passing a degrader and a trigger system [3,4], in a high density cryogenic gaseous target, filled with ${ }^{3} \mathrm{He}$ as a first step, ${ }^{4} \mathrm{He}$ in a second phase. A similar target was recently installed in DA $\Phi$ NE, for the SIDDHARTA [5] experiment and our group will take advantage of the gained experience.

\section{3. - Analysis of the KLOE data searching for $K^{-}{ }^{4} \mathrm{He}$ interactions}

Presently, we are performing dedicated Monte Carlo simulations, to study the performance of the AMADEUS setup. In parallel, we are analysing the existing KLOE data. Indeed the KLOE drift chamber is mainly filled with ${ }^{4} \mathrm{He}(90 \%$ helium $10 \%$ isobutane) and the analysis of KLOE Monte Carlo showed, that about $0.1 \%$ of kaons from DA $\Phi$ NE should stop in the inner volume of the drift chamber. This represents a unique opportunity to check the reconstruction capability for $\Sigma$ and $\Lambda$ particles and for studying the hadronic interactions of $K^{-}$in such an active target. Up to now, data for a total luminosity of $1.8 \mathrm{fb}^{-1}$ were analysed from a sample of 2005 KLOE data [6]. An excellent result was already achieved in reconstructing the $\Lambda(1116)$ invariant mass, with a statistical error of $3 \mathrm{keV}$; the systematics, being presently under evaluation.

We Acknowledge the support of the European Community-Research Infrastructure Integrating Activity "Study of Strongly Interacting Matter" (acronym Hadron-Physics2, Grant Agreement no. 227431) under the Seventh Framework Programme of EU.

\section{REFERENCES}

[1] The AMAdeus Collaboration, Letter of Intent, http://www.Inf.infn.it/ esperimenti/siddharta/LOI_AMDEUS_March2006.pdf.

[2] The AMAdEUS Collaboration, LNF preprint, LNF07/24(IR) (2007).

[3] Bazzi M., Corradi G., Piscicchia K., Romero Vidal A., Scordo A., Tagnani D. and Vasquez Doce O., AMADEUS Technical Note IR-2 04/10/2009.

[4] Bazzi M., Corradi G., Piscicchia K., Romero Vidal A., Scordo A., Tagnani D. and Vasquez Doce O., AMADEUS Technical Note IR-1 29/09/2009.

[5] Bazzi M. et al., Phys. Lett. B, 681 (2009) 310.

[6] Cargnelli M., Petrascu C. and Vazquez Doce O. (Kloe K-Charged Group), KLOE memo 337 (2007). 